\title{
Evaluation of therapeutic potential of intraperitoneal ozone gas in combination with insulin above cranial and spinal neuropathy in rats with diabetes mellitus
}

\author{
Karademir $\mathrm{M}^{1}$, Sonmez $\mathrm{MA}^{2}$, Akcilar R ${ }^{3}$, Kocak E${ }^{4}$, Yay A ${ }^{5}$, Eser $\mathrm{O}^{2}$ \\ Cumhuriyet University School of Medicine, Department of Neurosurgery, Sivas, Turkey. krdmr58@gmail.com
}

\begin{abstract}
OBJECTIVE: This study aimed to investigate the therapeutic effect of ozone in combination with insulin on cranial and spinal neuropathy in rats with diabetes mellitus (DM).

MATERIALS AND METHODS: Sixty adult male Sprague Dawley rats were randomly divided into the following six groups $(n=10)$ : control $(C)$, ozone $(O)$, diabetic $(D)$, ozone-treated diabetic (DO), insulin-treated diabetic (DI), and ozone, insulin-treated diabetic (DOI). DM was induced by a single intraperitoneal (ip) streptozotocin injection (60 mg/kg), followed by $3 \mathrm{IU}$ (ip) insulin administration for 60 days. Next, $1.1 \mathrm{mg} / \mathrm{kg}$ (50 $\mathrm{\mu g} / \mathrm{ml}$ ) ozone was administered to the $\mathrm{O}, \mathrm{DO}$, and $\mathrm{DO}$ groups for 60 days. After inducing diabetes, the total oxidant status (TOS) and total antioxidant status (TAS) were measured; the oxidative stress index (OSI) was calculated. The brain and spinal cord tissues were obtained for histopathological evaluation. This cross sectional study was conducted in Dumlupinar University Laboratory Animals Research Center e.g 11.03.2015 - 15.05.2015.

RESULTS: TAS was higher in the DO, DI, and DOI groups than in the D group. TOS and OSI were lower in the $\mathrm{DO}, \mathrm{DI}$, and $\mathrm{DOI}$ groups than in the D group. Little pathological alterations with degenerated axons and vascular congestion were observed in the DO, DI, and DOI groups compared with the D group.

CONCLUSION: Ozone with insulin can stimulate the endogenous antioxidant defense mechanism in diabetic neuropathy, thereby preventing reactive oxygen species-induced damage and protecting against cranial and spinal neuropathies (Fig. 6, Ref. 29).

KEY WORDS: intraperitoneal ozone gas, cranial and spinal neuropathy, diabetes.
\end{abstract}

\section{Introduction}

Diabetes mellitus (DM) is a global public health problem with epidemic proportions, and $60-70 \%$ of affected individuals have associated neurovascular complications that affect multiple organ systems (1). The most common and clinically significant neuropathies of DM include uremic neuropathy, peripheral neuropathy, and cardiac autonomic neuropathy. These conditions have serious effects on an individual's quality of life and significantly increase the risk for morbidity and mortality.

Chronic hyperglycemia, hypertension, dyslipidemia, insulin resistance, and uremia may be key factors that contribute to the pathogenesis of DM. In addition, the diseased state leads to changes in gene expression, molecular transport, inflammation, and oxidative stress.

${ }^{1}$ Cumhuriyet University School of Medicine, Department of Neurosurgery, Sivas, Turkey, ${ }^{2}$ Balikesir University School of Medicine, Department of Neurosurgery, Balikesir, Turkey, ${ }^{3}$ Dumlupinar University School of Medicine, Department of Physiology, Kütahya, Turkey, ${ }^{4}$ Dumlupinar University School of Medicine, Department of Biochemistry, Kütahya, Turkey, and ${ }^{5}$ Erciyes University School of Medicine, Department of Histology and Embryology, Kayseri, Turkey

Address for correspondence: M. Karademir, MD, Cumhuriyet University School of Medicine, Department of Neurosurgery, 58140 Sivas, Turkey . Phone: +90.542 .5600117 , Fax: +90.346 .2191010$
Diabetic neuropathy is a multifactorial disease that can either directly or indirectly affect the complete physiological system mainly because of the presence of the altered glucose metabolism and oxidative stress mechanism in DM. Moreover, neural tissues are affected, causing malfunction of the central and peripheral nerves (2).

About two-thirds of both patients with insulin-dependent DM and those with non-insulin-dependent DM had subclinical or clinical findings of peripheral neuropathy. Moreover, approximately half of the patients with diabetes had symmetric polyneuropathy, one-fourth had carpal tunnel syndrome, nearly $5 \%$ had autonomic neuropathy, and $1 \%$ had asymmetric proximal neuropathy. The occurrence of neuropathy correlated with the duration of DM, poor glycemic control, and presence of retinopathy or nephropathy (3-5). Multiple approaches have been attempted for treating diabetic neuropathy and other complications of diabetes; however, the success rate is poor. Glucose control, symptomatic treatment (using tricyclic antidepressants, carbamazepine, gabapentin, mexiletene, and more recently, pregabalin and cymbalta) are some of the attempted experimental approaches. Most attempts, such as autoimmune therapies, metabolic treatments, nerve growth factors, neuroactive steroids, acetyl-L-carnitine, $\alpha$-lipoic acid, $\gamma$-linoleic acid, neuroactive steroids, progesterone, testosterone, docosahexaenoic acid, and reproductive steroid derivations, have been used for preventing diabetic peripheral neuropathy (6). 
Ozone, a highly reactive gas, is produced in human white cells as part of the regulation of infection (7). Ozone has been used as a therapeutic agent for treating different diseases (8) and has a strong oxidizing power and good antiseptic, disinfectant, and antiviral properties (9). Furthermore, ozone has been proposed as an immunomodulator and activator of cellular metabolism. A recent study confirmed the long-term anti-inflammatory effects of ozone with an apparently low toxicity (10). Ozone therapy stimulates the endogenous antioxidant system activities in endotoxic and septic shock models (11). Moreover, the protective effects of ozone are known as ozone oxidative preconditioning (ozone OP). Ozone OP stimulates the antioxidant endogenous systems along with the regulation of reactive oxygen species (ROS) (12).

The clinical applications of ozone therapy have been extensively assessed in animal experiments and studies as part of diabetes research. The current study used a streptozotocin (STZ)induced diabetes rat model because it has significant similarities with patients with diabetes in terms of functional, pathological, and biochemical changes (13). The pathological basis for diabetic neuropathy remains controversial despite extensive research efforts.

There are a number of studies that suggest that both vascular and metabolic disorders may be responsible for peripheral nerve pathology in DM (14). An overview of these mechanisms approaches the different clinical presentations of diabetic neuropathies and helps to understand various experimental therapeutic trials.

We also explored the possibility that the mechanisms of ozone therapy upon cranial and spinal tissue neuropathies in rats with diabetes is attributable to the activation of antioxidant protection systems. Thus, this study aimed to evaluate the effectiveness of ozone therapy against diabetes and its complications in rats.

\section{Materials and methods}

All experimental animal protocols were carried out according to the National Institutes of Health Guidelines for the Care and Use of Laboratory Animals (NIH Publication No. 85-23) and were approved by the Dumlupinar University Local Ethical Committee (approval number: 2015. 03. 01; approval date: 11.03.2015). Sixty adult male Sprague Dawley rats weighing $265 \pm 15 \mathrm{~g}$ (mean $\pm \mathrm{SD}$ ) were used. All subjects were maintained in a 12-h light/dark cycle environment (lights on 7:00-19:00 h) at $22 \pm 1{ }^{\circ} \mathrm{C}$ and $50 \%$ humidity and were kept in transparent plastic cages $(42 \times 26 \times 15$ $\mathrm{cm}$ ). The rats had access to standard chow pellets (Altromin 1324, Lage, Germany) and tap water ad libitum throughout the study.

The animals were randomly divided into the following six groups $(n=10)$ : control $(C)$, ozone $(O)$, diabetic (D), ozone-treated diabetic (DO), insulin-treated diabetic (DI), and ozone, insulintreated diabetic (DOI). Diabetes was induced by a single intraperitoneal (ip) injection of freshly prepared STZ (Sigma-Aldrich Co., Taufkirchen, Germany) solution $(60 \mathrm{mg} / \mathrm{kg}$ body weight in $0.09 \mathrm{M}$ citrate buffer; $\mathrm{pH} 4.8$ ) in the D, DO, DI, and DOI groups. The animals in the $\mathrm{C}$ and $\mathrm{O}$ groups received similar volumes of the delivery vehicle. Hyperglycemia was confirmed at $48 \mathrm{~h}$ after STZ injection by measuring tail vein blood glucose levels using a glucometer (Accu-Chek; Roche Diagnostics Co., Mannheim,
Germany). Only rats with mean plasma glucose levels of $>300$ $\mathrm{mg} / \mathrm{dl}$ were classified as having diabetes. Blood glucose levels of all the rats were measured before and after the experimental procedures. Ozone was generated using an ozone generator (Dr. J. Hänsler Ozonosan GmbH, Iffezheim, Germany). A $50 \mu \mathrm{g} /$ $\mathrm{ml}$ concentration of ozone was administered to the $\mathrm{O}, \mathrm{DO}$, and DOI groups $(1.1 \mathrm{mg} / \mathrm{kg}$ ip; 1 -min injection period once a day for 60 days) (5). This dosage and schedule of ozone was previously used in other researches (15). This ozone dose achieves oxidative preconditioning without appreciable toxicity (16). Insulin (3 IU) (Novo Nordisk Co., Bagsvaerd, Denmark) was intraperitoneally administered to the DI and DOI groups (in $1 \mathrm{ml}$ saline, once a day for 60 days). In addition, $1 \mathrm{ml}$ saline was injected in the $\mathrm{C}, \mathrm{O}, \mathrm{D}$, and DO groups every day for 60 days.

The rats were sacrificed at the end of the study providing overdose anesthesia with ketamine/xylazine (90 and $10 \mathrm{mg} / \mathrm{kg}$, respectively; ip). The brain and spinal cord tissues were obtained for histopathological evaluation. The abdomens of the rats were immediately opened via a midline incision under anesthesia, and blood samples from the abdominal aorta were collected in citrated tubes. The blood samples were then centrifuged at $1500 \times \mathrm{g}$ for 10 min, and plasma was then separated and stored at $-80^{\circ} \mathrm{C}$.

\section{Biochemical analysis}

The tissue homogenates were prepared to estimate total oxidation status (TOS) and total antioxidant status (TAS). Approximately $1 \mathrm{~g}$ of tissue sample was mixed with $9 \mathrm{ml}$ of working solution (50 mmol phosphate buffer; $\mathrm{pH}$ 7.40) and homogenized using a mechanical homogenizer (Analytic Jena Speedmill Plus, Germany). The mixture was then centrifuged at $3000 \mathrm{rpm}$ at $5 \mathrm{~min}$ always with the same $\mathrm{G}$ force, and the supernatant was used to measure TAS and TOS.

\section{Total antioxidant status measurement (TAS)}

Tissue TAS concentrations were measured using the Beckman Coulter AU680 analyzer (Beckman Coulter, Miami, FL, USA) with a commercial TAS kit (Rel Assay Diagnostic, Turkey). The reduced ABTS (2,2'-azinobis 3-ethylbenzo-thiazoline-6-sulfonate) molecules were oxidized to ABTS using hydrogen peroxide in an acidic medium (acetate buffer $30 \mathrm{mmol} / \mathrm{L} ; \mathrm{pH} 3.6$ ). In the acetate buffer solution, the concentrated ABTS molecules remained stable for a longer period of time. While it was diluted with a more concentrated acetate buffer solution at a higher $\mathrm{pH}$ (acetate buffer 0.4 $\mathrm{mol} / \mathrm{l}$; pH 5.8), the color was spontaneously and slowly bleached. Antioxidants present in the sample accelerated the bleaching rate to a degree proportional to their concentrations.

\section{Total oxidant status measurement (TOS)}

Tissue TOS concentrations were measured using the Beckman Coulter AU680 analyzer (Beckman Coulter) with a commercial TOS kit (Rel Assay Diagnostic, Turkey). Through this kit, oxidants present in the sample oxidized the ferrous ion-o-dianisidine complex to ferric ions. The oxidation reaction was enhanced by glycerol molecules, which were abundant in the reaction medium. The ferric ions developed a colored complex with xylenol orange 
636-641

in an acidic medium. The color intensity was related to the total quantity of oxidant molecules present in the sample.

\section{Calculation of oxidative stress index (OSI)}

The ratio of TOS to TAS is referred to as the oxidative stress index (OSI). OSI was calculated according to the formula: OSI $=$ TOS ( $\mu \mathrm{mol} \mathrm{H}_{2} \mathrm{O}_{2}$ equiv/l)/TAS (mmol Trolox equiv/l) (6).

\section{Histopathological evaluation}

For histopathological evaluation, the rats from control $(\mathrm{C}),(\mathrm{O}$ and experimental (D, DI, DO, and DOI) groups were decapitated. Harvested brain and spinal cord tissues were fixed in $10 \%$ formalin for $24 \mathrm{~h}$, then dehydrated in a graded ethanol series, cleared in xylene, and embedded in paraffin. Then, $5-\mu \mathrm{m}$-thick serial sections were placed on poly-L-lysine slides. Samples from all the groups were stained with hematoxylin-eosin (H\&E) stain to obtain a morphological overview of the tissue and its structure. After staining, the sections were observed without knowing which rat was which, under the Olympus BX-51 light microscope (Olympus BX-51, Japan), and photographs were taken.

\section{Statistical analyses}

One-way ANOVA and post hoc Tukey's test were used for comparisons among the groups. All results are expressed as mean $\pm \mathrm{SD}$, and $\mathrm{p}<0.05$ were considered to be statistically significant.

\section{Results}

\section{Oxidative stress findings}

Total oxidant status measurement (TOS) levels were significantly increased in the Diabetic (D), ozone-treated diabetic (DO), insulin-treated diabetic (DI), and ozone, insulin-treated diabetic (DOI) groups compared with the Control (C) group $(\mathrm{p}<0.05)$. In contrast, total oxidant status measurement (TOS) levels were significantly decreased in the ozone-treated diabetic (DO), insulintreated diabetic (DI), and ozone, insulin-treated diabetic (DOI) groups compared with the Diabetic (D) group $(\mathrm{p}<0.05)$. However, the differences observed with regard to total antioxidant status measurement (TAS) and total oxidant status measurement (TOS)

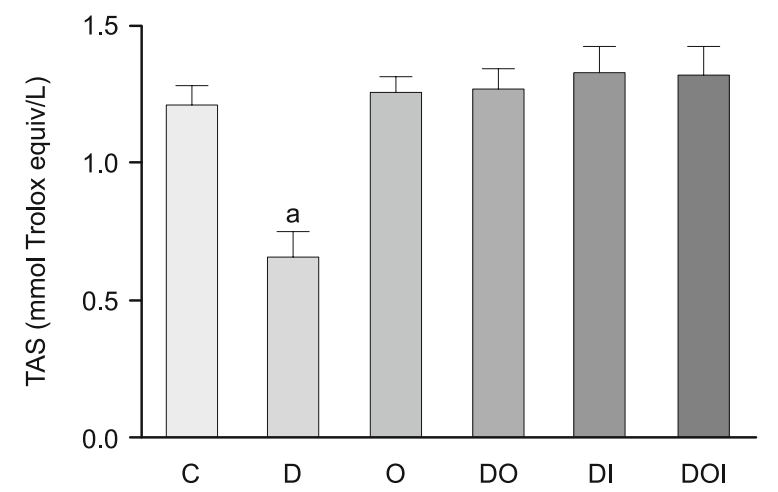

Fig. 1. Total Antioxidant Status (TAS) Plasma Levels. ${ }^{\text {a }}$ Statistically different when compared to the control $(p<0.05)$.

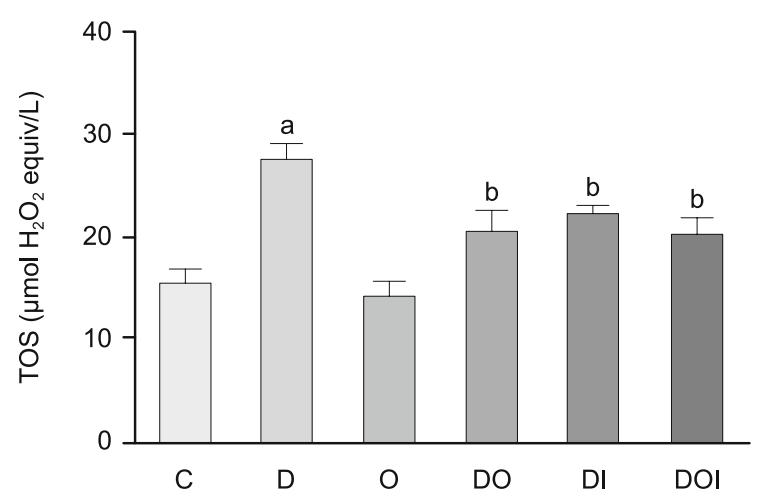

Fig. 2. Total Oxidant Status (TOS) Levels. ${ }^{a}$ Statistically different when compared to the control $(p<0.05),{ }^{\text {b }}$ Statistically different when compared both control and Diabetic group $(p<0.05)$.

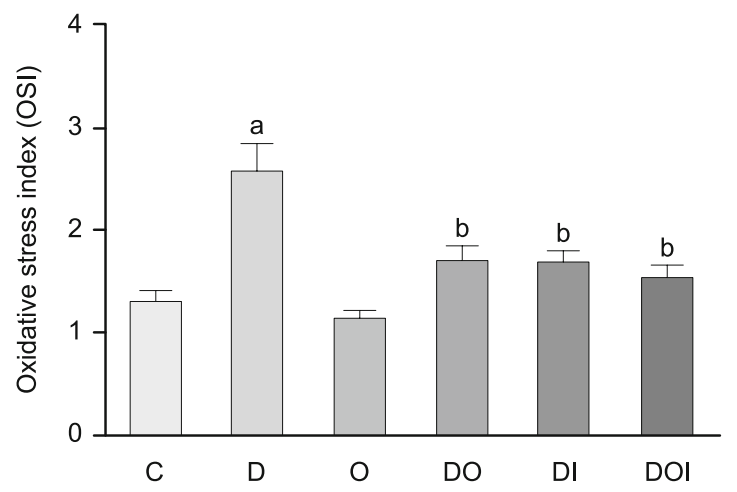

Fig. 3. Oxidative Stress Index (OSI). ${ }^{\text {a }}$ Statistically different when compared to the control $(p<0.05),{ }^{\text {b }}$ Statistically different when compared both control and Diabetic group $(p<0.05)$.

levels among the ozone-treated diabetic (DO), insulin-treated diabetic (DI), and ozone, insulin-treated diabetic (DOI) groups were not significant $(\mathrm{p}>0.05)$. Total antioxidant status (TAS) measurement levels were significantly higher in the insulin-treated diabetic (DI) and ozone, insulin-treated diabetic (DOI) groups than in the control group $(\mathrm{C})(\mathrm{p}<0.05)$. Moreover, total antioxidant status (TAS) measurement levels were significantly increased in the ozone-treated diabetic (DO), insulin-treated diabetic (DI), ozone, insulin-treated diabetic (DOI) groups compared with the Diabetic (D) group $(p<0.05)$. The oxidative stress index (OSI) levels were significantly decreased in the ozone-treated diabetic (DO), insulin-treated diabetic (DI), and ozone, insulin-treated diabetic (DOI) groups compared with the Diabetic (D) group $(\mathrm{p}<0.05)$. Total oxidant status measurement (TOS) and oxidative stress index (OSI) levels were significantly decreased in the ozone-treated diabetic (DO), insulin-treated diabetic (DI), and ozone, insulin-treated diabetic (DOI) groups compared with the Diabetic (D) group (p $<0.05$ ) (Figs 1, 2 and 3).

\section{Blood glucose levels}

As shown in Figure 4, higher blood glucose levels were observed in the D, DO, DI, and DOI groups than in the $\mathrm{C}$ and $\mathrm{O}$ 


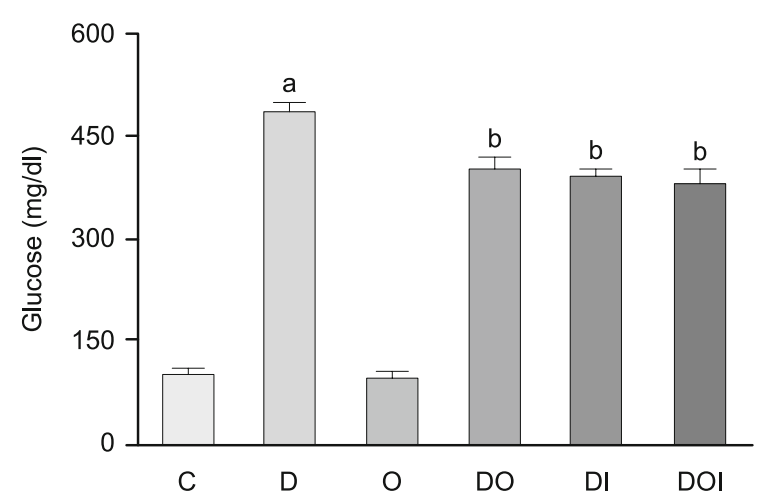

Fig. 4. Plasma Glucose Levels (mg/dl). a Statistically different when compared to the control $(p<0.05),{ }^{\text {b }}$ Statistically different when compared both control and Diabetic group $(p<0.05)$.
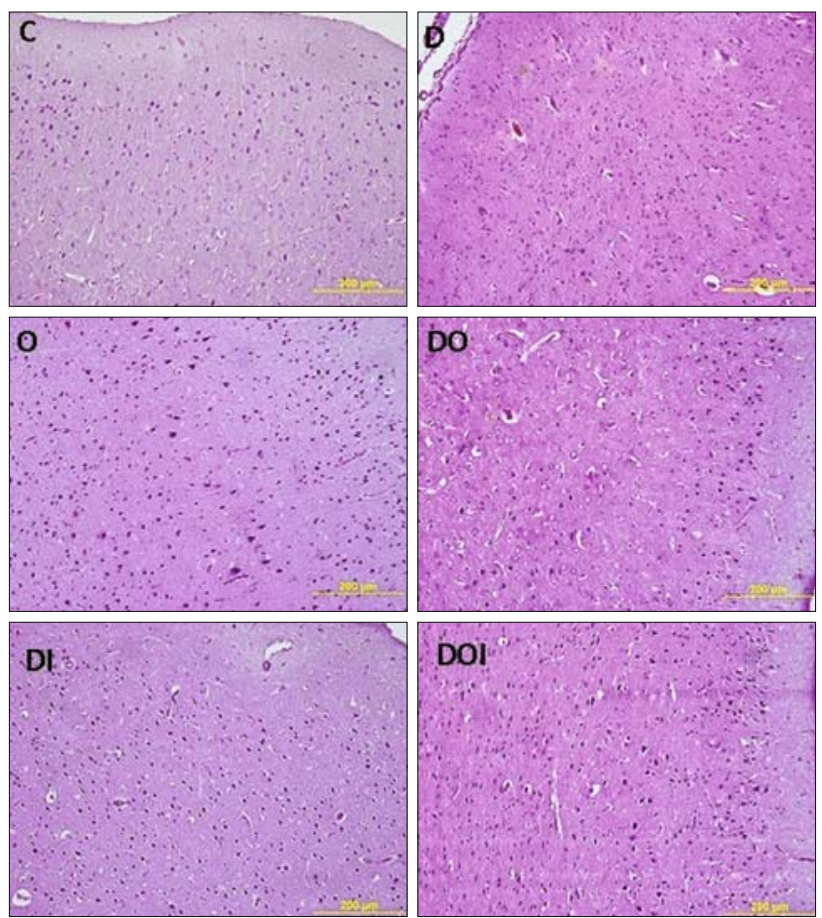

Fig. 5. Photomicrographs of rat brain tissues in cross section of each study group (H\&E staining). Original magnification, $\times 20$. C group; normal brain tissue structure in the control group. $D$ group; the brain was damaged after STZ administration. O group; normal histological architecture was observed. DO group; histological picture of STZ + Ozone group showed a significant amelioration of brain architecture. Histological picture showed a significant decrease in brain damage in the DI and DOI groups.

groups. Furthermore, the blood glucose levels were significantly lower in the DO, DI, and DOI groups than in the D group. The ozone-only treatment did not significantly change the blood glucose levels of the rats in the $\mathrm{O}$ group compared with those of the rats in the $\mathrm{C}$ group. In addition, blood glucose levels were significantly lower in the DI group than in the DO group. Similarly, blood glucose levels were significantly lower in the DOI group than in the DO and DI groups.
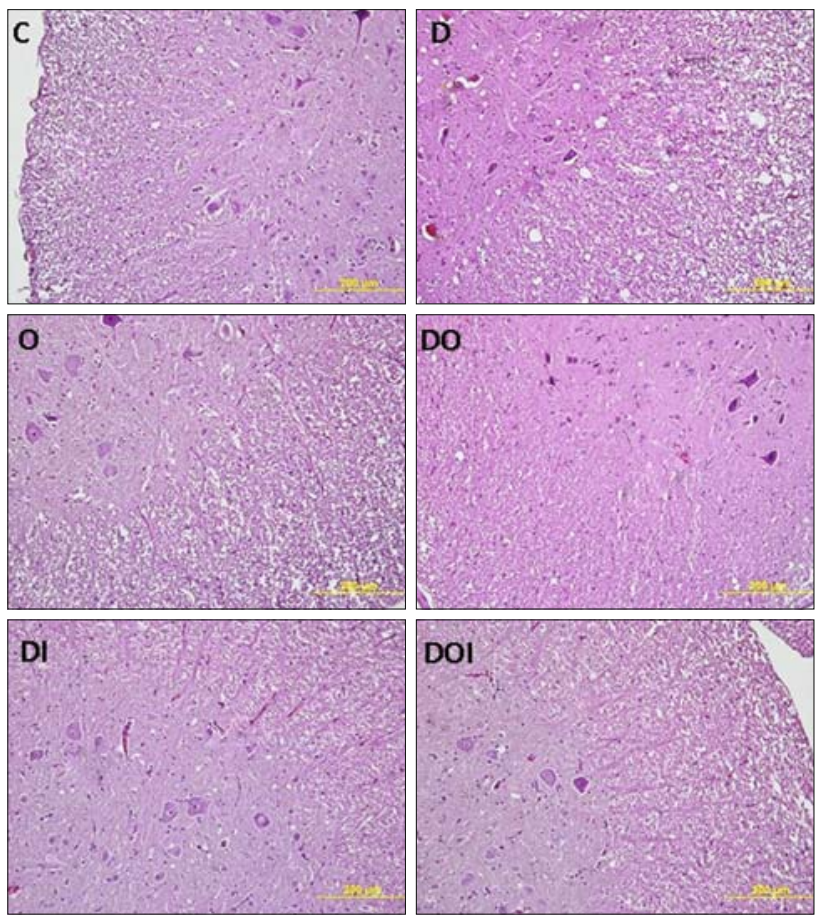

Fig. 6. Images are representatives of the H\&E-stained sections of the medulla spinalis from the experimental groups (H\&E staining). Original magnification, $\times 20$. C group; medulla spinalis structures were normal. The white matter contains myelinated nerve fibers and neuroglial cells. The gray matter contained neuronal cell bodies and their dendrites, along with central neuroglia. D group the medulla spinalis structure was damaged after STZ was administered. There was vascular congestion in both the gray and white matter. $O$ group; the general architecture of the medulla spinalis was structurally normal. DO group; ozone treatment apparently alleviated STZ-induced histopathological damages. DI group; insulin treatment improved the medulla spinalis histological architecture except the vascular congestion. DOI group; the histological appearance of the DOI group was similar to that of the DI group.

\section{Histopathological findings}

For histological evaluation, the H\&E dye technique was used to stain the nucleus and cytoplasm of the cells, which appeared blue and pink, respectively, as shown in Figures 5 and 6, respectively. Examination of the brain and spinal cord sections revealed normal histological architecture with cell bodies of nerves in the rats without diabetes. The spinal cord is organized into the outer part, white matter, and inner part. The gray matter contains nerve cell bodies and associated nerve fibers. Pathological changes in the vessels and neural cells were detected in the brain and spinal cord cortex of rats with diabetes. Microscopic examination of the brain and spinal cord samples showed degenerated axons in the white matter and vascular congestion in both the gray and white matter. The brain and spinal cord tissues from the O group showed a normal architecture, although few degenerated areas were observed. Moreover, the DO group showed few pathological alterations such as degenerated axons and vascular congestion. The histological appearance of the DI group was similar to that of the DOI group. In addition, the DOI group improved the architectures of both the brain and spinal tissues compared with the D group. 


\section{Discussion}

The most common metabolic disorder caused by total or relative insulin insufficiency is DM. DM is characterized by hyperglycemia and impaired metabolism of carbohydrates, fats, and proteins (17). Moreover, high blood glucose levels that result from insulin resistance in peripheral tissues or impaired insulin synthesis in the pancreas are also common in patients with diabetes (18). High blood glucose levels are also associated with the generation of reactive oxygen species (ROS) and consequent oxidative damage to the liver, kidneys, and pancreas. In addition, the implication of oxidative stress in DM pathogenesis has been proposed to alter antioxidant enzymes and the formation of lipid peroxides (19). Various pathways are involved in the pathogenesis of diabetic neuropathy and degeneration. The imported pathways included mechanisms responsible for advanced glycation, oxidative stress, and inflammation. Oxidative stress and inflammation play a crucial role in the development and progression of late-stage complications during diabetes (20).

DM-induced diabetic neuropathy is associated with oxidative stress. The potential therapeutic effects of ozone therapy were assessed in rats with STZ-induced diabetes (21). Treatment with either insulin or ozone therapy significantly reversed the effects of DM. Furthermore, a combination of both insulin and ozone therapy further reversed the effects of DM compared with monotherapy. Thus, ozone administration in rats with DM reduced oxidative stress marker levels and improved antioxidant enzyme activities, especially when the rats were treated with a combination of ozone and insulin (6). This initial result suggested that ozone therapy is useful for treating patients with diabetic neuropathy by specifically mediating antioxidative responses.

Ozone is a gas molecule comprising dynamically unstable three oxygen atoms. The gas is colorless and has a peculiar smell. The half-life of ozone is $40 \mathrm{~min}$ at $20^{\circ} \mathrm{C}$ and approximately $140 \mathrm{~min}$ at $0{ }^{\circ} \mathrm{C}$. Ozone has ability to stimulate 2,3-diphosphoglycerate that in turn induces an elevation in oxygen supply to the tissues, leading to increased production of enzymes, which act as free radical scavengers and cell wall savers, such as superoxide dismutase, glutathione peroxidase, and catalase. An increase in the output of prostacycline, which has a vasodilator property, improves blood microcirculation and tissue oxygen supply $(23,24)$. It is quite clear that ozone has multiple therapeutic properties because it acts as an antimicrobial and anti-inflammatory agent and is a modulator of the antioxidant defense system and regulator of apoptosis. ROS also play a major role in the pathogenesis of diabetic neuropathy.

There is evidence that hyperglycemia decreases enzyme activities of superoxide dismutase (SOD) and glutathione (GSH) synthesis, presumably via glycation (25). However, it is currently not possible to define how ozone treatment decreases hyperglycemia. In contrast, patients with diabetes have low antioxidant defenses, both with enzymatic (SOD, catalase, GSH) and non-enzymatic (vitamin C, E, or A, free radical scavengers or "total antioxidant capacity") factors (26). All these results proposed that protective effects of ozone on antioxidant endogenous defenses improve glucose metabolism. This study showed that ozone treatment de- creased blood glucose levels in rats with diabetes compared with control rats. These results confirmed that the protective effects of ozone in diabetic neuropathy are associated with the oxidant-antioxidant mechanism. High doses of ozone increased the oxidant enzyme levels and decreased the antioxidant enzyme levels. The ozone dose used is a major determinant of the effects of ozone on the oxidant-antioxidant balance (27).

Blood levels of diverse oxidant species could be measured one by one, but these tests are expensive, time-consuming, and require complex instrumentation. Nowadays, the evaluation of TOS levels is performed by evaluating serum lipid peroxidation levels. Protective enzymes, namely SOD, CAT, glutathione reductase, and GSH, react against the destructive actions of ROS, and these molecules compose TAS. The advantage of TAS measurement is that it could determine the antioxidant capacity of all antioxidants in a biological sample (28). Substantially, the ratio of TOS to TAS is named as OSI, which is a more precious parameter than TAS or TOS level alone to reflect oxidative status (29). Erken et al also demonstrated that medical ozone therapy given by rectal insufflation reduced TOS and OSI levels and partially prevented diabetic neuropathy in rats (6). In line with this study, we noted the neuroprotective effects of ozone as reflected by a decrease in TOS and OSI levels.

In the present study, we found that TOS and OSI levels were significantly high in the D group. Although TAS levels were lower in the D group and higher in the D, DI, and DOI groups, there were no significant changes detected in TAS values between the DO, DI, and DOI groups.

\section{Conclusion}

There are a numerous different murine models of DM but none of the models completely represents just the pathophysiology of the disease. We believe that more preclinical animal research needs to improve on different levels to yield best predictions for human DM patients. In conclusion, ozone may increase the endogenous antioxidant defense mechanism in rats, thereby protecting them from cranial and spinal tissue neuropathies. Moreover, the combination of ozone and insulin therapy has better prospectus for treating DM.

\section{References}

1. Witzel I, Jelinek HF, Khalaf K, Lee S, Khandoker AH, Alsafar H. Identifying common genetic risk factors of diabetic neuropathies. Front Endocrinol (Lausanne) 2015; 6: 88.

2. Brownlee M. Biochemistry and molecular cell biology of diabetic complications. Nature 2001; 414: 813-820.

3. Dyck PH, Kratz KM, Karnes JL, Litchy WJ, Klein R, Pach JM et al. The prevalence by staged severity of various types of diabetic neuropathy, retinopathy, and nephropathy in a population-based cohort: the Rochester diabetic neuropathy study. Neurology 1993; 43: 817-824.

4. Rosoklija GB, Dwork AJ, Younger DS, Karlikaya G, Latov N, Hays AP. Local activation of the complement system in endoneurial microvessels of diabetic neuropathy. Acta Neuropathol 2000; 99: 55-62. 
5. Dyck PJ, Windebank AJ. Diabetic and nondiabetic lumbosacral radiculoplexus neuropathies: new insights into pathophysiology and treatment. Muscle Nerve 2002; 25: 477-491.

6. Erken HA, Genç O, Erken G, Ayada C, Gündoğdu G, Doğan H. Ozone partially prevents diabetic neuropathy in rats. Exp Clin Endocrinol Diabetes. 2015; 123: 101-105.

7. Wright ES, Dziedzic D, Wheeler CS. Cellular, biochemical and functional effects of ozone: new research and perspectives on ozone health effects. Toxicol Lett 1990; 51: 125-145.

8. Hernandez FA, Calunga JL, Turrent J, Mene'ndez CS, Montenegro PA. Ozone therapy effects on biomarkers and lung function in asthma. Arch Med Res 2005; 36: 549-554.

9. Wells KH, Latino J, Gavalchin J, Poiesz BJ. Inactivation of human immunodeficiency virus type 1 by ozone in vitro. Blood 1991; 78: 1882-1890.

10. Chang JD, Lu HS, Chang YF, Wang D. Ameliorative effect of ozone on cytokine production in mice injected with human rheumatoid arthritis synovial fibroblast cells. Rheumatol Int 2007; 26: 142-151.

11. Zamora ZB, Borrego A, López OY, Delgado R, González R, Menéndez $\mathbf{S}$ et al. Effects of ozone oxidative preconditioning on TNF-alpha release and antioxidant-prooxidant intracellular balance in mice during endotoxic shock. Mediators Inflamm 2005; 24: 16-22.

12. Bocci V. Ozone as Janus: this controversial gas can be either toxic or medically useful. Mediators Inflamm 2004; 13: 3.

13. Yagihashi S. Nerve structural defects in diabetic neuropathy: do animals exhibit similar changes? Neurosci Res Commun 1997; 21: 25-52.

14. Pasnoor M, Dimachkie MM, Kluding P, Barohn RJ. Diabetic neuropathy part 1: overview and symmetric phenotypes. Neurol Clin 2013; 31: 425-445.

15. Morsy MD, Hassan WN, Zalat SI. Improvement of renal oxidative stress markers after ozone administration in diabetic nephropathy in rats. Diabeteol Metabol Syndr 2010; 2: 29-35.

16. León OS, Menéndez S, Merino N, Castillo R, Sam S, Pérez L et al. Ozone oxidative preconditioning: a protection against cellular damage by free radicals. Mediators Inflamm 1998; 7: 289-294.

17. Pooja VS, Samanta K. Hypoglycemic activity of methanolic extract of Tectona grandis Linn. Root in alloxan induced diabetic rats. J Appl Pharm Sci 2011; 4: 106-109.
18. De Silva DD, Rapior S, Hyde KD, Bahkali AH. Medicinal mushrooms in prevention and control of diabetes mellitus. Fungal Divers 2014; 56: $1-29$.

19. Ceriello A, Motz E. Is oxidative stress the pathogenic mechanism underlying insulin resistance, diabetes, and cardiovascular disease? The common soil hypothesis revisited. Arterioscler Thromb Vasc Biol 2004; 24: 816-823.

20. Schalkwijk CG, Poland DC, van Dijk W, Kok A, Emeis JJ, Dräger AM. Plasma concentration of C-reactive protein is increased in type I diabetic patients without clinical macroangiopathy and correlates with markers of endothelial dysfunction: evidence for chronic inflammation. Diabetologia 1999; 42: 351-357.

21. Bocci V, Zanardi I, Michaeli V, Travagli V. Mechanisms of action and chemical-biological interactions between ozone and body compartments: a critical appraisal of the different administration routes. Curr Drug Ther 2009; 4: 159-173.

22. Elvis AM, Ekta JS. Ozone therapy. A clinical review. J Nat Sci Biol Med 2011; 2: 66-70.

23. Bocci VA. Scientific and medical aspects of ozone therapy. State of the art. Arch Med Res 2006; 37: 425-435.

24. Chen H, Xing B, Liu X, Zhan B, Zhou J, Zhu H et al. Ozone oxidative preconditioning inhibits inflammation and apoptosis in a rat model of renal ischemia/reperfusion injury. Eur J Pharmacol 2008; 581: 306-314.

25. West IC. Radicals and oxidative stress in diabetes. Diabet Med 2000; 17: 171-180.

26. Yoshida K, Hirokawa J, Tagani S, Kawakani Y, Urata Y, Kondo T. Weakened cellular scavenging activity against oxidative stress in diabetes mellitus: regulation of glutathione synthesis and efflux. Diabetologia 1995; 38: 201-210.

27. Bocci V, Borrelli E, Travagli V, Zanardi I. The ozone paradox: ozone is a strong oxidant as well as a medical drug. J Dent 2008; 36: 104-116.

28. Kusano C, Ferrari B. Total antioxidant capacity: a biomarker in biomedical and nutritional studies. J Cell Mol Biol 2008; 7: 1-15.

29. Sancaktutar AA, Bodakci MN, Hatipoglu NK, Soylemez H, Basarili K, Turkcu G. The protective effects of pomegranate extracts against renal ischemia-reperfusion injury in male rats. Urol Ann 2014; 6: 29-46.

Received May 23, 2018. Accepted June 26, 2018. 